\title{
Eustachian tube catheterization: fundamental skill for competent otolaryngologists
}

Keywords: eustachian tube catheterization, eustachian tube dysfunction

Abbreviations: ETC, eustachian tube catheterization; ETD, eustachian tube dysfunction; LPR, laryngopharyngeal reflux; GERD, gastroesophageal reflux disease; OME, otitis media with effusion

\section{Editorial}

\section{Diagnosis \& treatment of mechanical obstruction of eustachian tube}

'Hearing loss', 'ear fullness', 'dizziness' (vertigo), 'tinnitus', and 'headache' (otalgia, migraine). These are the symptoms that are commonly reported by patients at my otolaryngology outpatient clinic. On hearing any combination of these symptoms, what do you think is the first thing I consider? I always first consider the state of the middle ear and the Eustachian tube. In my opinion, Eustachian tube catheterization (ETC) must be practiced with the proper technique in order to diagnose or rule out Eustachian tube dysfunction. ${ }^{1}$

\section{Eustachian tube catheterization: my preferred technique}

My 20+ years of medical experience have taught me the value of excellent research. I am a clinician who inherited Eustachian tube catheterization through apprenticeship, and I feel an urge to preserve this simple yet delicate diagnostic and therapeutic procedure. I strongly maintain that ETC is one of the fundamental and essential skills for a competent otolaryngologist and can be of both diagnostic and therapeutic value to ideally normalize middle ear cavity pressure with a perfectly equal balance between both ears. ${ }^{2}$

I would like to introduce the method which I perform in my clinic using devices such as the 'Eustachian tube catheter' and the 'Toynbee diagnostic tube.' The procedure is more than a century old and was previously routinely performed to assess the patency of the Eustachian tube.

This technique is called the 'Rosenmüller method. ${ }^{3}$ The nasal tip is tilted upward with the finger of the operator until the anterior nares are raised level with the floor of the nose. The tip of the catheter is inserted into the nose, then passed first upward and along the floor of the nasal cavity till it touches the posterior pharyngeal wall. The tip of the catheter is in the nasopharynx. At this point, the tip is rotated $90^{\circ}$ laterally so that it touches the Rosenmüller fossa behind the Eustachian tube in the lateral wall of the nasopharynx. The tip is pulled forward slightly, and passed over the gentle elevation of the cartilaginous opening of the torus of the auditory tube (or torus tubarius), and rotated $45^{\circ}$ superior-laterally again so that it enters the opening of the Eustachian tube. A ventilator is attached to a port at the external end of the catheter through a tube. Air is pulsed into the catheter and, if the Eustachian tube is patent, it can be heard rushing into the ear. The operator can confirm the state and quality of this sound by auscultation with a Toynbee diagnostic tube (Figure 1).

\author{
Volume II Issue I - 2019 \\ Hee-Young Kim \\ Department of Otolaryngology, Kim ENT Clinic, Republic of \\ Korea
}

Correspondence: Hee-Young Kim, Department of Otolaryngology, Kim ENT Clinic, 2nd fl. I I 9, Jangseungbaegi-ro Dongjak-gu, Seoul, 06935, Republic of Korea, Tel +82 02855 754I,Email kheeyoung@gmail.com

Received: January 03, 2019 | Published: January 04, 2019

The medial and lateral rotations that complicate the procedure are designed to avoid the tubal elevation located behind the tubal opening because the catheter tip cannot be visualized once inserted into the nose. This is a blind procedure, with only a metal ring located on the proximal end of the catheter to indicate the direction of the catheter tip. However, novice practitioners may perform the procedure under endoscopic guidance to enable visualization of the opening of the Eustachian tube. Liquids and sprays are sometimes injected into the ear through the catheter. Bougies can also be passed into the Eustachian tube in the same manner as the catheter or, if flexible, they may be passed through the catheter. As the tip of the bougie passes into the bony portion of the canal, the constriction of the isthmus can be felt $2.5 \mathrm{~cm}$ up from the canal's mouth. The bougie should not be passed farther than $3 \mathrm{~cm}$ into the Eustachian tube, because if the tympanum is entered, the ossicles may be injured. It is important to remember that this method only tests the patency of the Eustachian tube and does not reflect its function.

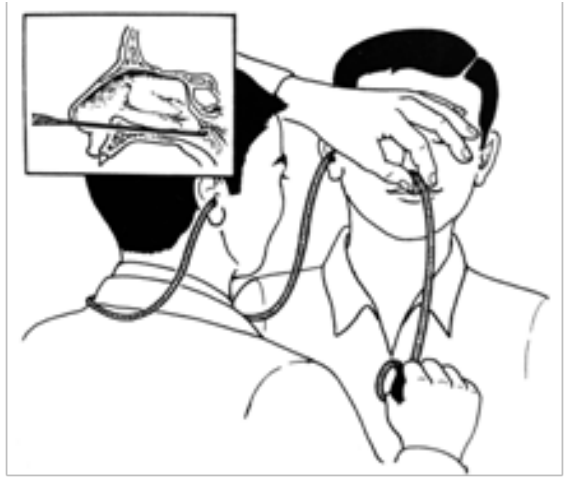

Figure I Eustachian tube catheterization with a Toynbee diagnostic tube.

Reciprocal causal relationship between
laryngopharyngeal reflux and eustachian tube
dysfunction

Especially in cases of Eustachian tube dysfunction (ETD) with accompanying nausea, vomiting, vertigo, diaphoresis, there is a distinct reciprocal causal relationship with laryngopharyngeal reflux (LPR) or gastroesophageal reflux disease (GERD) which results in a cycle of ETD and laryngopharyngeal/gastroesophageal reflux. ${ }^{4}$ 
When the onset of nausea and vertigo is sudden and severe, diagnosis of ETD is more easily established. If the proper treatment is instituted promptly, relief from symptoms is striking and dramatic. Eustachian tube dysfunction cases with insidious onset are most likely to be misdiagnosed. In these instances, because the primary symptoms are gastrointestinal complaints, patients are likely to be subject to various treatments for diseases of the digestive system, over long periods of time. Gastrointestinal symptoms like nausea and vomiting related to vertigo can also be regarded as symptoms of LPR or GERD. The symptoms and diseases associated with LPR or GERD should be included in the multiple symptoms and diseases which may originate from ETD.

Eustachian tube dysfunction can be caused by LPR. Because reflux material from the stomach can reach the nasopharynx and the Eustachian tubes, it can block the tubes directly or cause inflammation and adhesion, causing the tubes to collapse. ${ }^{5}$

A study on middle ear effusions (MEEs) obtained adenoidal tissue biopsies from patients undergoing simultaneous tympanostomy tube placement and adenoidectomy. In the control group, adenoid specimens were taken during adenoidectomy ( $+/$ - tonsillectomy) from children with no history of otitis media with effusion (OME). The adenoid tissues were immunohistochemically analyzed to confirm the presence of pepsinogen. This study demonstrates that LPR plays an important role in the pathogenesis of OME and establishes that gastric reflux can reach the middle ear through the nasopharynx and Eustachian tube which can cause OME.

\section{Eustachian tube catheterization: the present}

Although ETD has long been recognized as the principal cause of a variety of symptoms, ${ }^{6}$ it has received only scant attention in both the literature and the clinic. The Eustachian tube catheter and the Toynbee diagnostic tube which I use in my own clinic have fallen out of favor with most otolaryngologists. Although endorsed by the FDA, these devices are often relegated to the history books or treated as relics which belong in a museum.

\section{Eustachian tube catheter and Toynbee diagnostic tube are endorsed by the U.S. food and drug administration}

According to the Code of Federal Regulations Title 21, which was revised on April 1, 2018, by the U.S. FDA, the 'auscultation tube' is labeled as 'Sec. 874.1925 Toynbee diagnostic tube' and defined as follows: 'a listening device intended to determine the degree of openness of the Eustachian tube. ${ }^{77}$ The Eustachian tube catheter can be found in 'Sec. 874.4175 Nasopharyngeal catheter', and is described as follows: 'a device consisting of a bougie of filiform catheter that is intended for use in probing or dilating the Eustachian tube. This generic type of device includes Eustachian catheters. ${ }^{8}$

\section{Eustachian tube catheterization: the history (Figure 2)}

In 1724, Eustachian tube catheterization was first described by Edmé Gilles Guyot. Much later, in 1850, Joseph Toynbee invented the 'auscultation tube', which he called an 'otoscope.'

It would be no exaggeration to say that the history of otology is in line with the development of ETC. The literature and materials related to ETC from 1724 to 1861 are presented in 'The history of otology' (2015) published by Albert Mudry, Kugler Publications. There is some conflict in terms of differing interpretations of the historical significance of the different materials. Between 1724 and 1861, the standard for instruments, technique, and history of ETC were not yet firmly established. However, the history and development of this procedure have a significant impact on the treatment of patients in the clinic today.

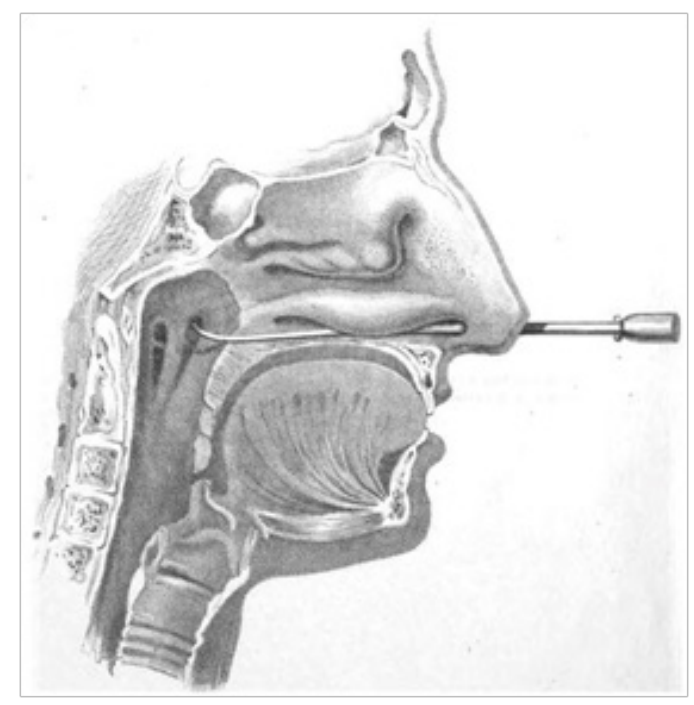

Figure 2 Image: Cross-section of a face with the catheter inserted through Eustachian tube.

\section{I have subdivided the history of ETC from I724 to I $86 \mathrm{I}$ into the following seven themes:}

i. Beginning of Eustachian tube catheterization for therapeutic reasons (1724)

ii. Evaluation of the patency of the Eustachian tube (1825)

iii. Recognition of the normal Eustachian tube as a prerequisite for normal hearing (1836)

iv. Therapeutic test of inflation of the Eustachian tube (1836

v. Eustachian tube catheterization for diagnostic reasons (1838)

vi. Eustachian tube catheterization as the first step before therapeutic artificial perforation of the tympanic membrane (1843)

vii. Toynbee diagnostic tube used with Eustachian tube catheter (1861)

About 110 years passed after ETC was first described by Edmé Gilles Guyot in 1724 before it was recognized by Nicolas Deleau the Younger (in 1836) that a normal Eustachian tube was a prerequisite for normal hearing. Deleau also described at least one patient who presented the three symptoms of dizziness, tinnitus, and hearing impairment, which were later defined as Menière's disease. ${ }^{9}$ He treated the patient by catheterizing the Eustachian tube and administering an air douche, which ameliorated symptoms. ${ }^{6}$

It is the time to revive the spirit and achievements of these historical trailblazers in the field of Eustachian tube catheterization. One of the most important reasons for assessing tubal function is the need to make a differential diagnosis in a patients who have an intact tympanic membrane without evidence of otitis media but who has symptoms that might be related to ETD (otalgia, snapping or popping in the ear, fluctuating hearing loss, tinnitus, or vertigo). ${ }^{10}$ Eustachian tube catheterization is clearly of both diagnostic and therapeutic 
value. Patients with classic ETD symptoms as well as gastrointestinal symptoms should undergo therapeutic inflation of the Eustachian tubes as the first step of a thorough clinical investigation.

\section{Eustachian tube catheterization: the future}

Eustachian tube catheterization should also feature in the up and coming 'Digital Health Age.' The history of Eustachian tube catheterization is still ongoing.

Finally, I'd like to conclude this article with a quote from Dr. F.W. Merica ${ }^{11}$ who published 'Vertigo Due to Obstruction of The Eustachian Tubes', Journal of American Medical Association in 1942 :

"Many patients suffer unnecessarily the distressing symptoms of vertigo, nausea, and vomiting, sometimes for long periods, because their physicians fail to recognize the cause and to institute the simple procedure of mechanical inflation of the Eustachian tubes which would bring them relief."

\section{Funding details}

The author has no funding, financial relationship.

\section{Acknowledgments}

The author has no acknowledgments.

\section{Conflict of interests}

The author declares that there is no conflict of interest to disclose.

\section{References}

1. Kim HY. Diagnosis \& Treatment of Mechanical Obstruction of Eustachian Tube. J Otolaryngol ENT Res. 2014;1(1):000001.

2. Kim HY. Eustachian Tube Catheterization. J Otolaryngol ENT Res. 2015;3(2):00057.

3. Noh KT. Otolaryngology-Head and Neck Surgery. Ilchokak: Seoul, Republic of Korea; 1996

4. Kim HY. Reciprocal Causal Relationship between Laryngopharyngeal Reflux and Eustachian Tube Obstruction. J Otolaryngol ENT Res. 2015;2(6):00046.

5. Al-Saab F, Manoukian JJ, Al-Sabah B, et al. Linking laryngopharyngeal reflux to otitis media with effusion: pepsinogen study of adenoid tissue and middle ear fluid. J Otolaryngol Head Neck Surg. 2008;37(4):565571 .

6. Kim HY. The History of Eustachian Tube Catheterization. J Otolaryngol ENT Res. 2016;4(1):00084

7. Toynbee diagnostic tube. Code of Federal Regulations. 2018;21:448.

8. Nasopharyngeal catheter. Code of Federal Regulations. 2018;21:453.

9. Kim HY. Vertigo due to Eustachian Tube Dysfunction. Archives of Otorhinolaryngology-Head \& Neck Surgery. 2017;1(1):5.

10. Bluestone CD, Eustachian tube: Structure, function, and role in Middle-Ear Disease. 2e Kindle ed. People Medical Publishing HouseUSA: Raleigh, North Carolina; 2018.

11. Merica FW. Vertigo due to obstruction of the eustachian tubes: a clinical study based on one hundred and thirty-five cases. JAMA. 8(15):1282-1284. 